\title{
Echocardiographic evaluation of mitral valve regurgitation
}

\author{
Aiman Smer ${ }^{1}$, Navin C. Nanda ${ }^{2}$, Raziye E. Akdogan², Zeyad M. Elmarzouky², Subash Dulal ${ }^{2}$ \\ ${ }^{1} \mathrm{CHI}$-Health Creighton University School of Medicine, Omaha, NE 68124, USA. \\ 2Division of Cardiology, Department of Medicine, University of Alabama at Birmingham, Birmingham, AL 35233, USA.
}

Correspondence to: Dr. Navin C. Nanda, Division of Cardiology, Department of Medicine, University of Alabama at Birmingham, Birmingham, AL 35233, USA. E-mail: nanda@uab.edu

How to cite this article: Smer A, Nanda NC, Akdogan RE, Elmarzouky ZM, Dulal S. Echocardiographic evaluation of mitral valve regurgitation. Mini-invasive Surg 2020;4:52. http://dx.doi.org/10.20517/2574-1225.2020.36

Received: 15 Apr 2020 First Decision: 29 May 2020 Revised: 12 Jun 2020 Accepted: 2 Jul 2020 Published: 15 Aug 2020

Academic Editor: Azeem Latib Copy Editor: Cai-Hong Wang Production Editor: Jing Yu

\begin{abstract}
Echocardiography is the primary imaging modality for the evaluation of mitral valve regurgitation. A comprehensive assessment of mitral regurgitation using different echocardiographic techniques provides important information regarding the etiology and severity of mitral regurgitation and its consequences on cardiac function. In addition, echocardiography plays an important role in the management of patients with mitral regurgitation.
\end{abstract}

Keywords: Echocardiography, mitral valve, mitral regurgitation, severity

\section{INTRODUCTION}

Echocardiography is the gold standard diagnostic test for the evaluation of valvular heart disease, particularly mitral regurgitation $(\mathrm{MR})^{[1]}$. An accurate assessment of MR severity is vital for clinical decision-making. Two-dimensional (2D) and three-dimensional (3D) echocardiography are mainly used to identify the etiology and mechanism of MR, while Doppler techniques provide accurate assessment of MR severity. In addition, integration of other supportive findings such as size and function of the left ventricle (LV), coexistence of significant tricuspid regurgitation and pulmonary artery pressure play an important role in the decision-making process regarding the type and time of intervention for severe $\mathrm{MR}^{[2]}$. In this article, we will discuss in detail the role of echocardiography in the evaluation of MR. 


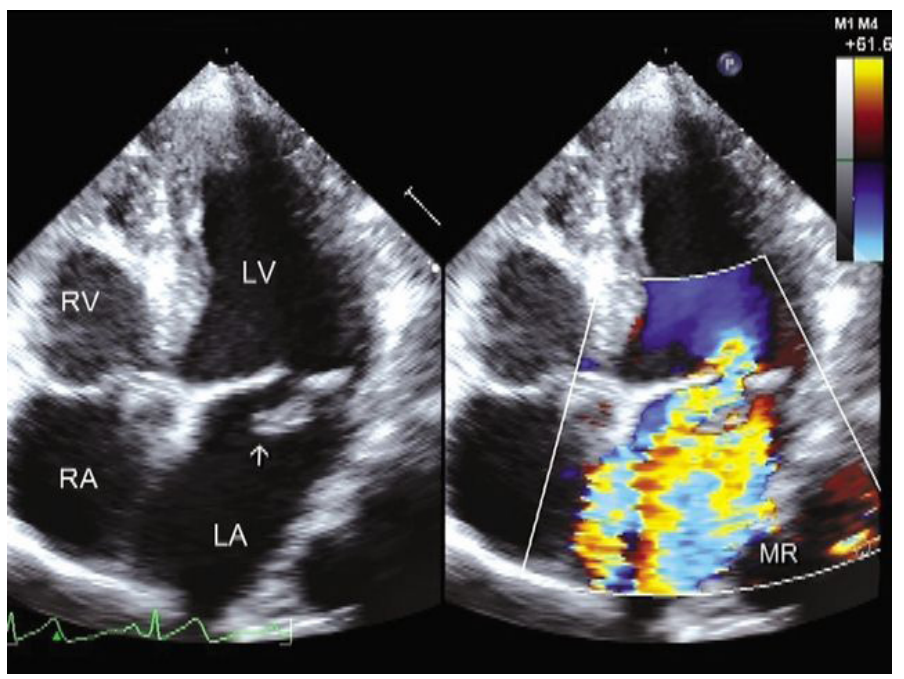

Figure 1. Two-dimensional transthoracic echocardiography demonstrates a myxomatous mitral valve and prolapse of posterior mitral leaflet (arrow) with severe MR. RV: right ventricle; RA: right atrium; LV: left ventricle; LA: left atrium; MR: mitral regurgitation. Reproduced with permission from Manjunath et al. ${ }^{[4]}$

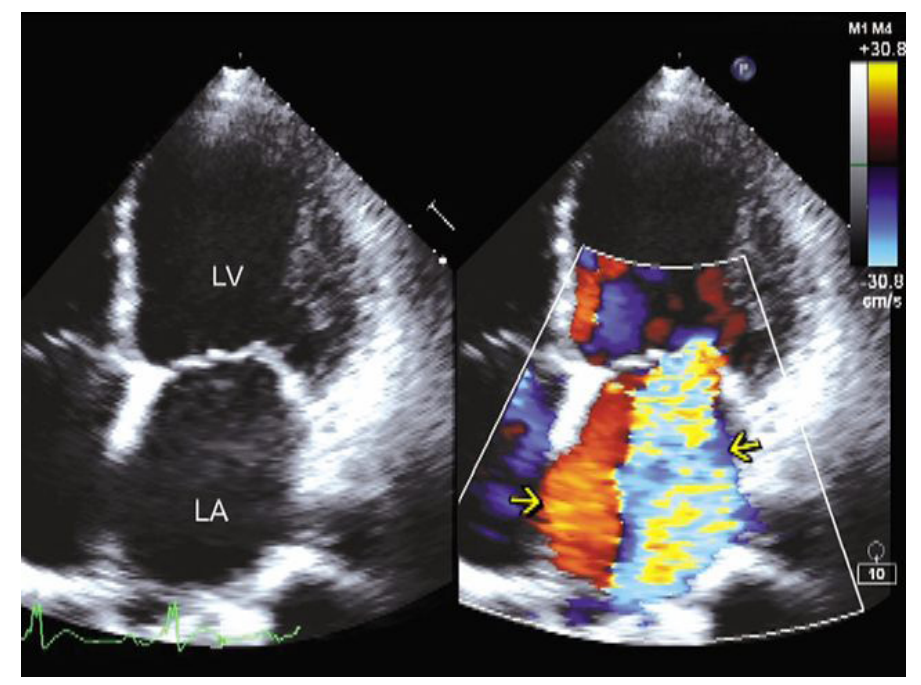

Figure 2. Two-dimensional transthoracic echocardiography in apical four-chamber view shows a dilated LV with mitral valve coaptation point displaced into the LV and severe MR. LV: left ventricle; LA: left atrium; MR: mitral regurgitation. Reproduced with permission from Manjunath et al. ${ }^{[4]}$

\section{ASSESSMENT OF ETIOLOGY AND MECHANISM OF MR}

Understanding the complex anatomy of the mitral valve (MV) is essential for accurate assessment of MR. The MV apparatus consists of mitral annulus, MV leaflets, chordae tendineae, papillary muscles and the underlying ventricular wall. Pathological abnormality of any one of these components can lead to MR [Figure 1] ${ }^{[3,4]}$. For instance, MR can occur due to primary (degenerative) MV disease affecting the MV leaflets and/or chordae tendineae, while secondary MR occurs due to a pathological process of the LV or left atrium (LA) [Figure 2] $]^{[4,5]}$. In case of ventricular disease, due to either ischemic or nonischemic cardiomyopathy, MR occurs due to regional or global remodeling of the LV, which causes lateral displacement of papillary muscles, resulting in annular dilation and leaflet tethering. However, there are some differences in the mechanism of MR in these two types of cardiomyopathy. The main mechanism for secondary MR in ischemic cardiomyopathy occurs due to inferior wall motion abnormalities, leading 


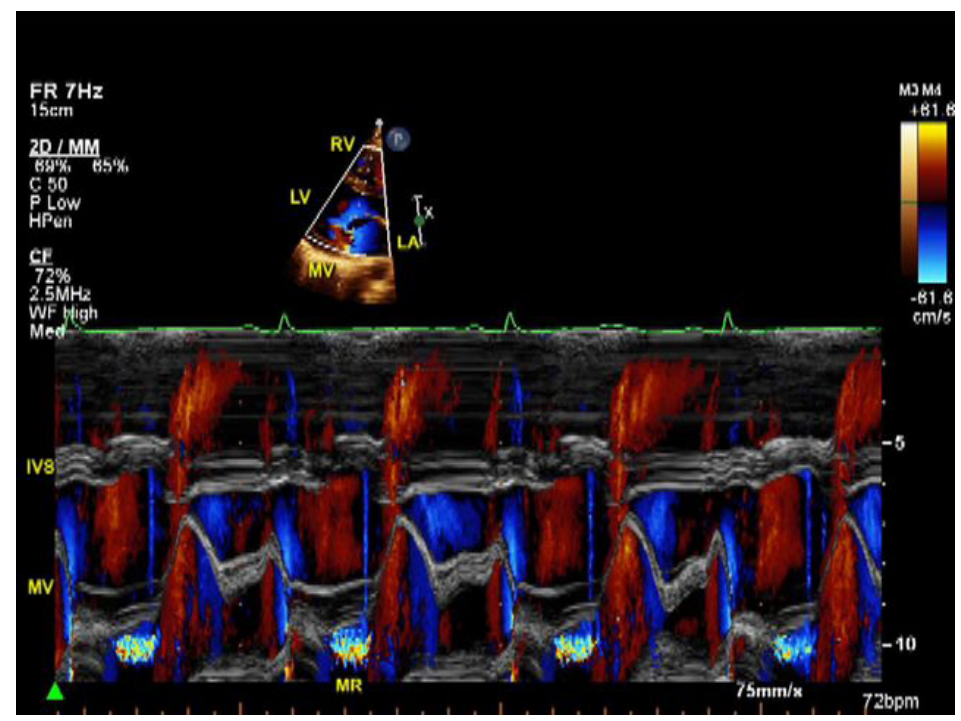

Figure 3. M-mode transthoracic echocardiogram in the parasternal long-axis view shows mid to late systolic MR in a patient with MV prolapse. IVS: interventricular septum; MR: mitral regurgitation; MV: mitral valve; LV: left ventricle; LA: left atrium; RV: right ventricle. Copyright with Aiman Smer

to systolic restriction and tethering of the posterior MV leaflet. This asymmetrical tenting pattern of the MV leaflets usually leads to posteriorly directed $\mathrm{MR}^{[6]}$. In contrast, non-ischemic secondary MR is usually associated with global wall motion abnormalities, leading to equal displacement of papillary muscles and annular dilation, which results in symmetrical tethering of both leaflets leading to central $\mathrm{MR}^{[7]}$.

Atrial fibrillation is another common cause of secondary MR, termed atrial MR. In patients with chronic atrial fibrillation, severe LA enlargement and annular dilation can lead to incomplete coaptation of the MV leaflets. Atrial remodeling of the mitral annulus can also lead to posterior displacement of the mitral annulus and tethering of the posterior MV leaflet ${ }^{[8]}$. In addition, myocardial dyssynchrony due to left bundle branch block or right ventricular pacing can potentially predispose to MR by a decrease in MV closing forces and dyssynchronous papillary muscle function ${ }^{[9]}$.

\section{M-MODE ECHOCARDIOGRAPHY}

M-mode was one of the earliest echocardiographic techniques to evaluate MV abnormalities ${ }^{[10]}$. The high temporal resolution of M-mode allows accurate diagnosis of the mechanism of MR in patients with MV prolapse and MR induced by hypertrophic obstructive cardiomyopathy. Adding color Doppler M-mode improves its diagnostic accuracy and helps determine whether MR is holosystolic or mid or late systolic [Figure 3]. This is an important aspect to consider since MR severity may be overestimated when using standard 2D/color Doppler imaging criteria of severity such as MR jet area, jet area/LA size ratio and proximal jet convergence and vena contracta $(\mathrm{VC})$ size, because they do not take into account the duration of MR.

\section{D ECHOCARDIOGRAPHY}

$2 \mathrm{D}$ transthoracic echocardiography (TTE) is the primary diagnostic test for the initial detection of MR and assessing its severity as well as evaluating the etiology and mechanism of MR [Table 1]. 2D echocardiography can easily differentiate between primary MR due to MV prolapse or ruptured chord versus secondary (functional) MR due to dilated LV. This anatomic assessment of the MV and LV in terms of morphology and function, can be particularly useful in determining whether percutaneous or surgical MV repair should be considered. The transthoracic parasternal long- and short-axis views of MV allow 
Table 1. Etiology and mechanism of mitral regurgitation

\begin{tabular}{ll}
\hline Etiology of mitral regurgitation & \multicolumn{1}{c}{ Mechanism of mitral regurgitation } \\
\hline Atrial fibrillation & Annular dilation, leaflet mal-coaptation \\
Acute ischemia & Papillary muscle dysfunction or rupture \\
Congenital or genetic disorders; Marfan syndrome, Ehlers-Danlos & Leaflet prolapse, cleft or rudimentary leaflets \\
syndrome, Down syndrome & \\
Endocarditis; infective and marantic & Leaflet perforation, mal-coaptation, chordal rupture \\
Drugs; fenfluramine and dexfenfluramine & Leaflets, chordae \\
Functional/secondary; dilated cardiomyopathy & Left ventricular remolding, papillary muscle displacement \\
& leading to leaflet tethering and annulus dilation \\
Hypertrophic obstructive cardiomyopathy & Systolic anterior motion of anterior mitral valve leaflet \\
Myxomatous degeneration (primary) & \\
(1) Barlow's disease & Leaflets prolapse \\
(2) Fibroelastic deficiency & Rupture chordae \\
Mitral annular calcifications & Annulus, leaflets \\
Rheumatic heart disease & Leaflets, chordae \\
Radiation & Leaflets, chordae \\
\hline
\end{tabular}
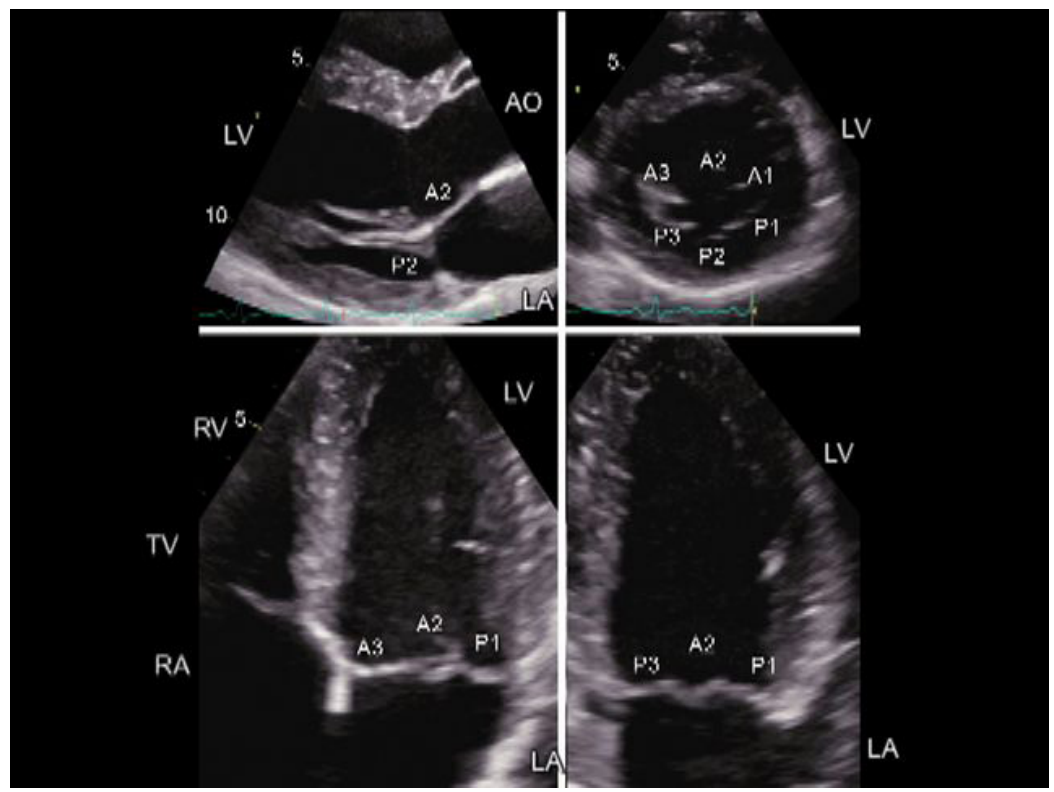

Figure 4. Mitral valve segment and scallop analysis with two-dimensional transthoracic echocardiography. Left upper panel: parasternal long-axis view depicting A2 segment and P2 scallop. Right upper panel: parasternal short-axis view permitting the assessment of A1, $A 2$ and $A 3$ segments and P1, P2 and P3 scallops. Left lower panel: apical four-chamber view showing A3, A2, and P1. Right lower panel: apical two-chamber view displaying P3, A2 and P1. RA: right atrium; RV: right ventricle; LV: left ventricle; LA: left atrium; TV: tricuspid valve; AO: aorta. Reproduced with permission from Pierard et al. ${ }^{[1]}$

direct visualization of mitral valve scallops and leaflet motion [Figure 4$]^{[11]} \cdot 2 \mathrm{D}$ echocardiography can also accurately diagnose rheumatic MR and endocarditis-induced MR.

2D transesophageal echocardiography (TEE) is indicated for evaluation of patients with MR in whom TTE is of poor quality or provides nondiagnostic information about the mechanism and severity of $\mathrm{MR}^{[2]}$. A jet area of 10-15 $\mathrm{cm}^{2}$ signifies severe MR. The proximity to the MV apparatus and 3D capabilities of TEE allow accurate assessment of MV abnormalities. In addition, TEE can provide additional information regarding the feasibility of percutaneous intervention and the likelihood of successful surgical repair. There are several TEE parameters required to assess the suitability of transcatheter edge-to-edge clip repair (MitraClip) for patients with severe chronic MR, who are deemed high surgical risk [Table 2] ${ }^{[12,13]}$ Echocardiographic features such as MV area, annular calcification and the number of scallops involved in MR can predict 
Table 2. Echocardiographic parameters for MitraClip feasibility

\begin{tabular}{|c|c|c|c|}
\hline & $\begin{array}{c}\text { Favorable } \\
\end{array}$ & Unfavorable & Contraindicated \\
\hline Etiology of MR & Myxomatous valve disease & $\begin{array}{l}\text { Severe annular dilation, }>50 \mathrm{~mm} \text { or } \\
\text { EROA }>70.8 \mathrm{~mm}^{2}\end{array}$ & $\begin{array}{l}\text { Rheumatic or endocarditis valve } \\
\text { disease }\end{array}$ \\
\hline Location of MR & Central, A2/P2 segments & Peripheral, $A 1 / P 1$ or $A 3 / P 3$ segments & Perforated mitral leaflets or clefts \\
\hline $\begin{array}{l}\text { Grasp zone } \\
\text { Calcification } \\
\text { Length }\end{array}$ & $\begin{array}{l}\text { None } \\
>10 \mathrm{~mm}\end{array}$ & $\begin{array}{l}\text { Mild } \\
7-10 \mathrm{~mm}\end{array}$ & $\begin{array}{l}\text { Moderate to severe } \\
<7 \mathrm{~mm}\end{array}$ \\
\hline $\begin{array}{l}\text { Mitral valve } \\
\text { Area } \\
\text { Gradient } \\
\text { Length of posterior leaflet } \\
\text { Leaflet mobility }\end{array}$ & $\begin{array}{l}>4 \mathrm{~cm}^{2} \\
<4 \mathrm{mmHg} \\
>10 \mathrm{~mm} \\
\text { Mobile }\end{array}$ & $\begin{array}{l}>3.5 \text { and }<4 \mathrm{~cm}^{2} \\
>4 \text { and }<5 \mathrm{mmHg} \\
7-10 \mathrm{~mm} \\
\text { Restricted motion }\end{array}$ & $\begin{array}{l}<3.5 \mathrm{~cm}^{2} \\
>5 \mathrm{mmHg} \\
<7 \mathrm{~mm} \\
\text { Immobile }\end{array}$ \\
\hline Primary MR & $\begin{array}{l}\text { Flail gap }<10 \mathrm{~mm} \\
\text { Flail width }<15 \mathrm{~mm}\end{array}$ & $\begin{array}{l}\text { Flail gap }>10 \mathrm{~mm} \\
\text { Flail width }>15 \mathrm{~mm}\end{array}$ & \\
\hline Secondary MR & $\begin{array}{l}\text { Coaptation depth }<11 \mathrm{~mm} \\
\text { Coaptation length }>2 \mathrm{~mm}\end{array}$ & $\begin{array}{l}\text { Coaptation depth }>11 \mathrm{~mm} \\
\text { Coaptation length }<2 \mathrm{~mm}\end{array}$ & \\
\hline
\end{tabular}

EROA: effective regurgitation orifice area; MR: mitral regurgitation

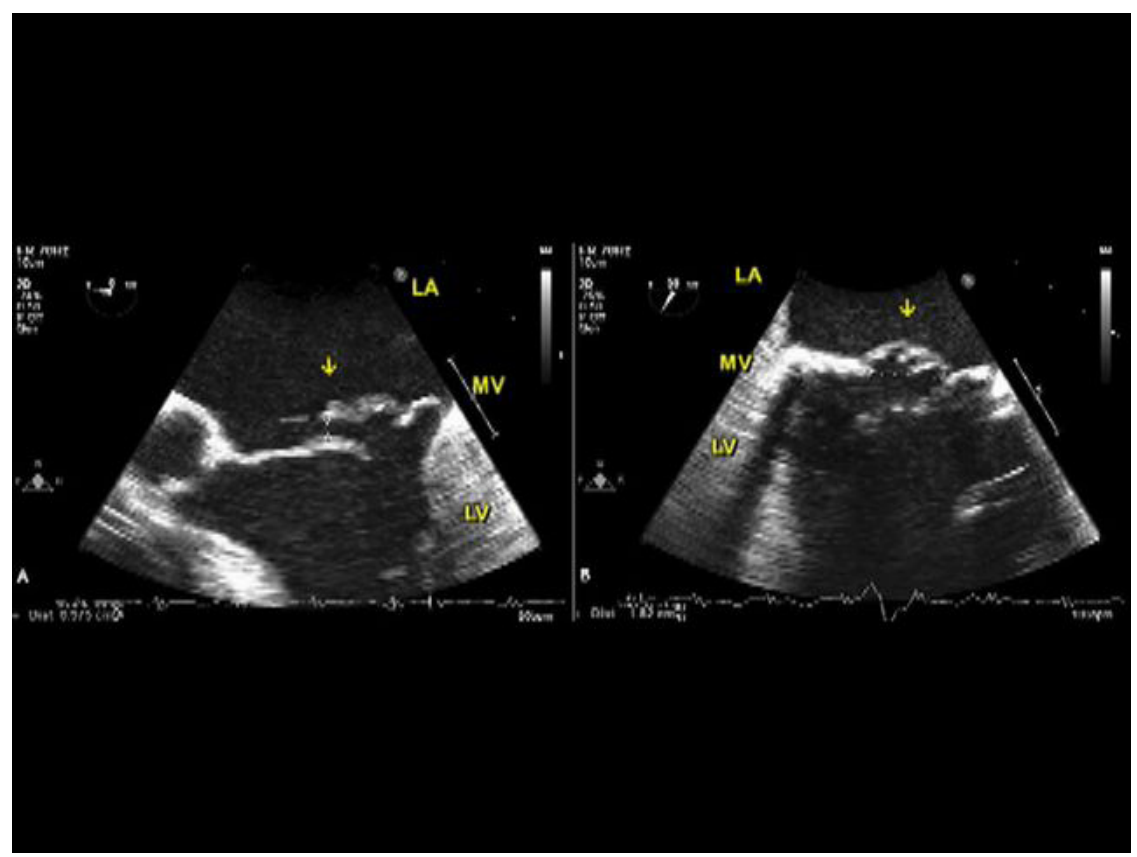

Figure 5. Two-dimensional transesophageal echocardiography demonstrates a flail MV (arrow) in five- and 2-chamber views. LV: left ventricle; LA: left atrium; MV: mitral valve. Copyright with Aiman Smer

successful MitraClip placement ${ }^{[14]}$. In primary MR, measurements of leaflet separation and flail gap and width are important for procedural success [Figure 5]. While in secondary MR, measurements of annular diameter and coaptation length and depth are essential to predict adequate leaflet grasping and successful repair [Figure 6]. In addition, TEE is essential to guide both surgical and percutaneous MV repair, immediately assess procedural success and identify potential complications.

\section{D ECHOCARDIOGRAPHY}

3D echocardiography either from a transthoracic or transesophageal approach can provide superb images of the MV apparatus. The ability of $3 \mathrm{D}$ imaging to visualize the MV from different $2 \mathrm{D}$ angles allows accurate assessment of $\mathrm{MR}^{[15]}$. A unique advantage of $3 \mathrm{D}$ TEE is the ability to provide an en face view of the MV from the LA perspective, which is similar to the surgeon's view in the operating room [Figure 7]. This view 


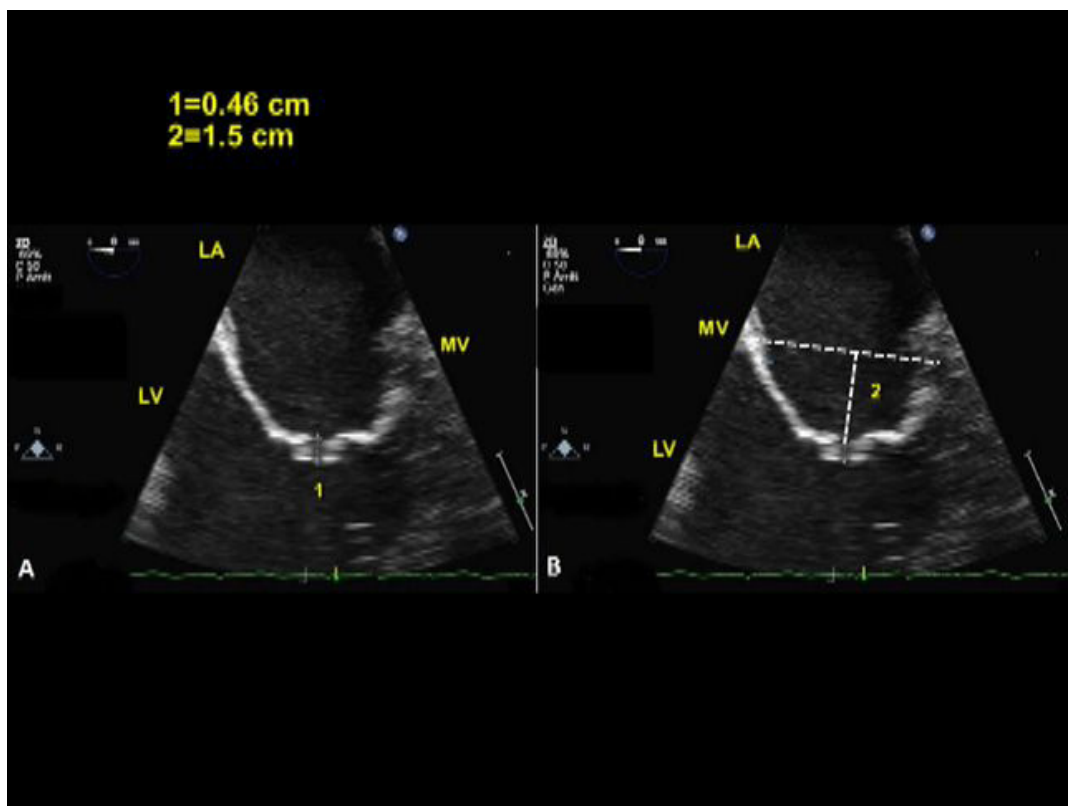

Figure 6. Two-dimensional transesophageal echocardiography demonstrates systolic non-coaptation gap (1) of the MV which is displaced into the LV. \#2 represents the perpendicular distance of the MV coaptation point from the MV annulus. LV: left ventricle; LA: left atrium; MV: mitral valve. Copyright with Aiman Smer

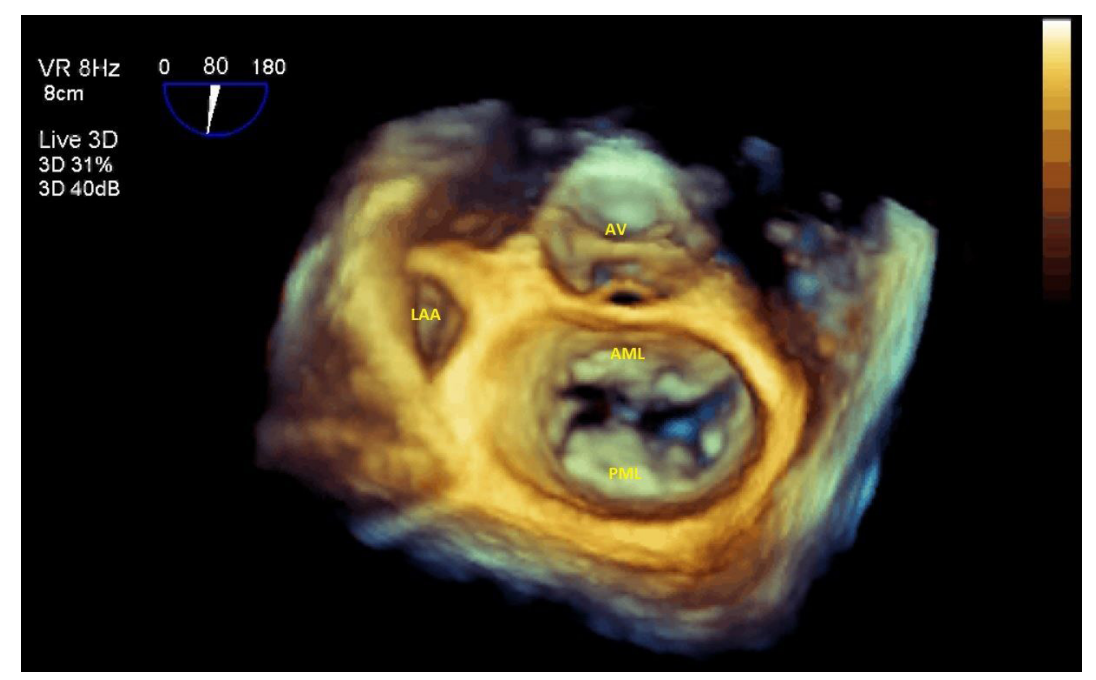

Figure 7. Live/real-time three-dimensional transesophageal echocardiography shows an en face view of the MV. MV: mitral valve; AML: anterior MV leaflet; AV: aortic valve; LAA: left atrial appendage; PML: posterior MV leaflet. Copyright with Aiman Smer

allows accurate localization of the involved MV leaflet or scallop in MR and also identify rare conditions such as MV cleft, which is very difficult to diagnose on $2 \mathrm{D}$ imaging. In addition, 3D echocardiography is especially useful in prosthetic MV regurgitation and guidance of percutaneous cardiac interventions. For instance, the use of real-time 3D TEE in MitraClip procedure is crucial for optimal trans-septal puncture and device placement.

In general, $2 \mathrm{D}$ and $3 \mathrm{D}$ echocardiography are mainly used to identify valve pathology and mechanism of MR. However, there are certain structural findings such as flail leaflet, ruptured papillary muscle, and large coaptation defect, which are specific for severe MR. In addition, dilated LV along with atrium with normal LV function suggests severe MR. 
Table 3. Grading the severity of mitral regurgitation

\begin{tabular}{|c|c|c|c|}
\hline & Mild & Moderate & Severe \\
\hline \multicolumn{4}{|l|}{ Qualitative parameters } \\
\hline MV morphology & Normal/abnormal & Normal/abnormal & Flail leaflet/chordal rupture \\
\hline Color flow Doppler of MR jet* & $<20 \%$ of LA size & $20 \%-40 \%$ of LA size & $>40 \%$ of $L A$ size \\
\hline \multicolumn{4}{|l|}{ Continuous wave Doppler } \\
\hline MR jet density & Faint & Dense & Dense \\
\hline MR jet contour & Parabolic & Parabolic & Early peaking-triangular \\
\hline Flow convergence zone* & No or small & Intermediate & Large \\
\hline \multicolumn{4}{|l|}{ Semi-quantitative parameters } \\
\hline Vena contracta & $<0.3 \mathrm{~cm}$ & $0.3-0.69 \mathrm{~cm}$ & $\geq 0.7 \mathrm{~cm}$ \\
\hline \multirow[t]{2}{*}{ Mitral valve inflow } & A-wave dominant & & E-wave dominant, $>1.2 \mathrm{~m} / \mathrm{s}$ \\
\hline & $\begin{array}{l}\text { Mitral to aortic TVI ratio } \\
<1 \mathrm{~m} / \mathrm{s}\end{array}$ & Mitral to aortic TVI ratio 1 to $1.4 \mathrm{~m} / \mathrm{s}$ & Mitral to aortic TVI > $1.4 \mathrm{~m} / \mathrm{s}$ \\
\hline Pulmonary veins flow & Systolic dominance & Normal or systolic blunting & Systolic flow reversal in $>1$ vein \\
\hline LA/LV size & Normal & Intermediate & $\begin{array}{l}\text { Enlarged, particularly with } \\
\text { normal LV function }\end{array}$ \\
\hline \multicolumn{4}{|l|}{ Quantitative parameters } \\
\hline $\begin{array}{l}\text { Effective regurgitant orifice area by } \\
\text { PISA or 3D color Doppler echo }\end{array}$ & $<0.2 \mathrm{~cm}^{2}$ & $\begin{array}{l}0.2-0.29 \mathrm{~cm}^{2} ; \text { Mild to moderate } \\
0.3-0.39 \mathrm{~cm}^{2} ; \text { Moderate to severe }\end{array}$ & $\geq 0.4 \mathrm{~cm}^{2}$ \\
\hline Regurgitant volume & $<30 \mathrm{~mL} /$ beat & $\begin{array}{l}\text { 30-44 mL/beat; Mild to moderate } \\
45-59 \mathrm{~mL} / \text { beat; Moderate to severe }\end{array}$ & $\geq 60 \mathrm{~mL} /$ beat \\
\hline Regurgitant fraction & $<30 \%$ & $\begin{array}{l}30 \%-39 \% \text {; Mild to moderate } \\
40 \%-49 \% \text {; Moderate to severe }\end{array}$ & $\geq 50 \%$ \\
\hline
\end{tabular}

MR: mitral regurgitation; MV: mitral valve; LA: left atrium; LV: left ventricle; TVI: time velocity integral. *At Nyquist limit between 50-70 $\mathrm{cm} / \mathrm{s}$. Color Doppler gain needs to be optimized

\section{ASSESSMENT OF SEVERITY OF MR}

Doppler echocardiography is the primary method for the detection and quantification of MR [Table 3] $]^{[16]}$. The density of the continuous wave Doppler signal of the MR envelop is a useful qualitative parameter of MR severity. In general, small, faint MR jets with little or no flow convergence zone indicate mild $\mathrm{MR}$, while large and dense jets with a large flow convergence or vena contracta are typically severe. A comprehensive color and spectral Doppler evaluation of MR using semi-quantitative and quantitative parameters should be performed when more than mild MR is suspected ${ }^{[17]}$.

An accurate assessment of MR severity is crucial for appropriate management and patient selection for interventional procedures. Given the limitations of standard echocardiographic methods in quantifying severe secondary MR, the concept of functional MR proportionality to the LV size has been proposed to accurately identify patients with clinically significant secondary $\mathrm{MR}^{[18]}$. If the regurgitant volumes of severe functional MR is still proportional to the LV size, the patient is less likely to benefit from MV interventions. On the other hand, when the regurgitant volumes become disproportional to the degree of LV dilation (MR is greater than expected for the given LV size), the patient is more likely to benefit from MV interventions ${ }^{[18]}$. This concept has gained more interest after the recent controversy about the results of the COAPT (Transcatheter Mitral-Valve Repair in Patients with Heart Failure) and MITRA-FR (Percutaneous Repair or Medical Treatment for Secondary Mitral Regurgitation) trials ${ }^{[19,20]}$. In these two large randomized trials on MitraClip placement for severe secondary MR, different echocardiographic eligibility criteria and definitions were used for MR severity ${ }^{[21]}$. The COAPT trial included patients with an effective regurgitant orifice area (EROA) of at least $0.3 \mathrm{~cm}^{2}$ and regurgitant volume (RVol) $>45 \mathrm{~mL} /$ beat, while MITRA-FR included patients with less severe functional MR, EROA of at least $0.2 \mathrm{~cm}^{2}$ and $\mathrm{RVol}>30 \mathrm{~mL} / \mathrm{beat}$. In addition, the COAPT trial included only patients with LV end-systolic dimension of $70 \mathrm{~mm}$ or less, while MITRA-FR did not have restrictions regarding LV size. Given the conflicting results of these two trials, further studies to test the concept of disproportionate functional MR are needed. Meanwhile, careful patient selection for MitraClip is essential to achieve favorable outcomes. 


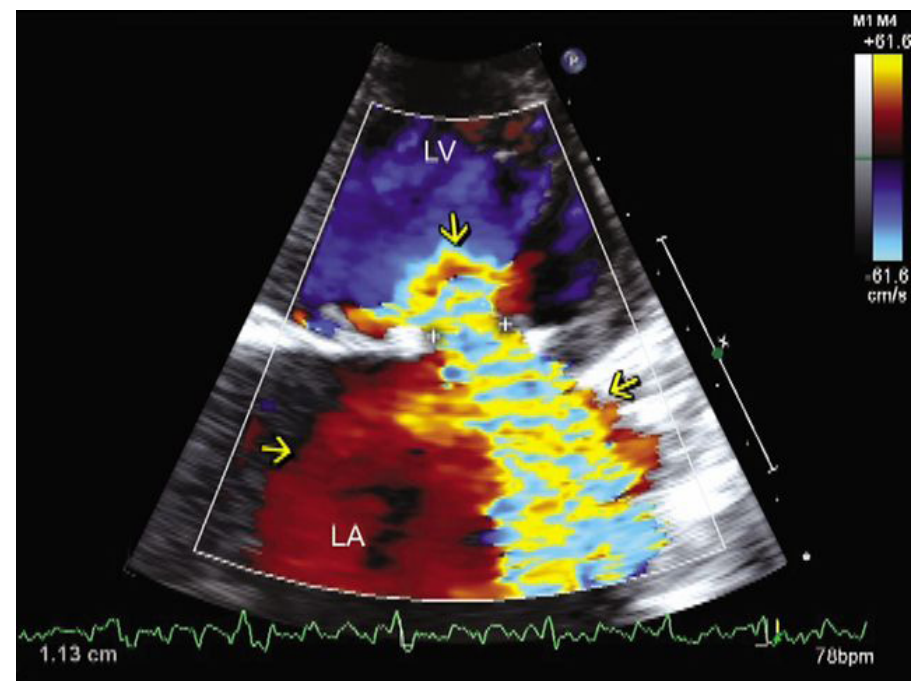

Figure 8. Two-dimensional transthoracic echocardiography in apical four-chamber view in a patient with severe MR. The vertical arrow points to a large flow acceleration, and the distance between the two + signs represents the vena contracta width, which measures 1.13 $\mathrm{cm}$, indicative of severe MR. Not only the turbulent flow signals (right arrow in LA) but also the accompanying laminar flow signals (red, left arrow in LA) moving in the same phase as the turbulent flow signals represent MR. Thus, MR flow signals practically completely fill the LA, indicative of torrential MR. MR severity would have been underestimated if the red laminar signals were not taken into account. LV: left ventricle; LA: left atrium; MR: mitral regurgitation. Reproduced with permission from Manjunath et al. ${ }^{[4]}$

\section{COLOR DOPPLER}

Color flow Doppler (CD) is commonly used for the detection and assessment of MR severity ${ }^{[22]}$. This technique allows visualization of MR and identifies several characteristics of the regurgitant jet including number of jets, site, direction and the three components of the regurgitant jet (flow convergence, vena contracta and jet area) [Figure 8$]^{[1,4]}$. Obtaining CD imaging of the MV in the parasternal short-axis view is important to localize the site of the MR jet. The spatial orientation of the regurgitant jet area within the LA during ventricular systole is proportional to the severity of $\mathrm{MR}^{[23]}$. On the basis of the percentage ratio of the color jet area to the LA, MR can be graded as mild, moderate or severe if the ratio is $<20 \%, 20 \%-40 \%$ or $>40 \%$, respectively ${ }^{[23]}$. However, it is important to understand that there are several technical and hemodynamic factors that can influence the relationship between the jet size and MR severity. For instance, using inappropriate Nyquist limit or color gain could over- or underestimate the color jet size. A lower Nyquist limit will exaggerate lower velocities, and thus make the MR jet appear larger, while reducing the color gain results in a smaller jet and vice versa [Figure 9] ${ }^{[4]}$. Thus, it is recommended to use a standard Nyquist limit between $50-70 \mathrm{~cm} / \mathrm{s}$ and optimize color gain to eliminate random lower flow velocity signals or color artifacts in the $\mathrm{LA}^{[1]}$. CD could be misleading in acute MR and in patients with hypotension or tachycardia. On the other hand, MR jet may appear larger in patients with elevated LV end-diastolic pressure due to high driving pressure across the MV, which can be seen in cases of significant aortic stenosis, LV outflow obstruction or uncontrolled hypertension. CD could also overestimate MR jet area when multiple jets are present ${ }^{[24]}$.

Eccentric MR jets are sometimes difficult to detect and appear smaller due to loss of energy when the regurgitant jet impinges the LA walls or the other leaflet, known as Coanda effect [Figure 10] ${ }^{[4,25]}$. This problem is often obviated when one takes into account the laminar (red/blue) flow signals moving in the same phase as the turbulent (mosaic colored) eccentric jet. Loss of energy from impingement results in low velocity and therefore laminar MR signals. Presence of eccentric jet generally indicates significant MR and should raise the suspicion of the possibility of underlying structural abnormalities such as torn chord or leaflet perforation. Careful evaluation of other echocardiographic parameters such as the presence of large 

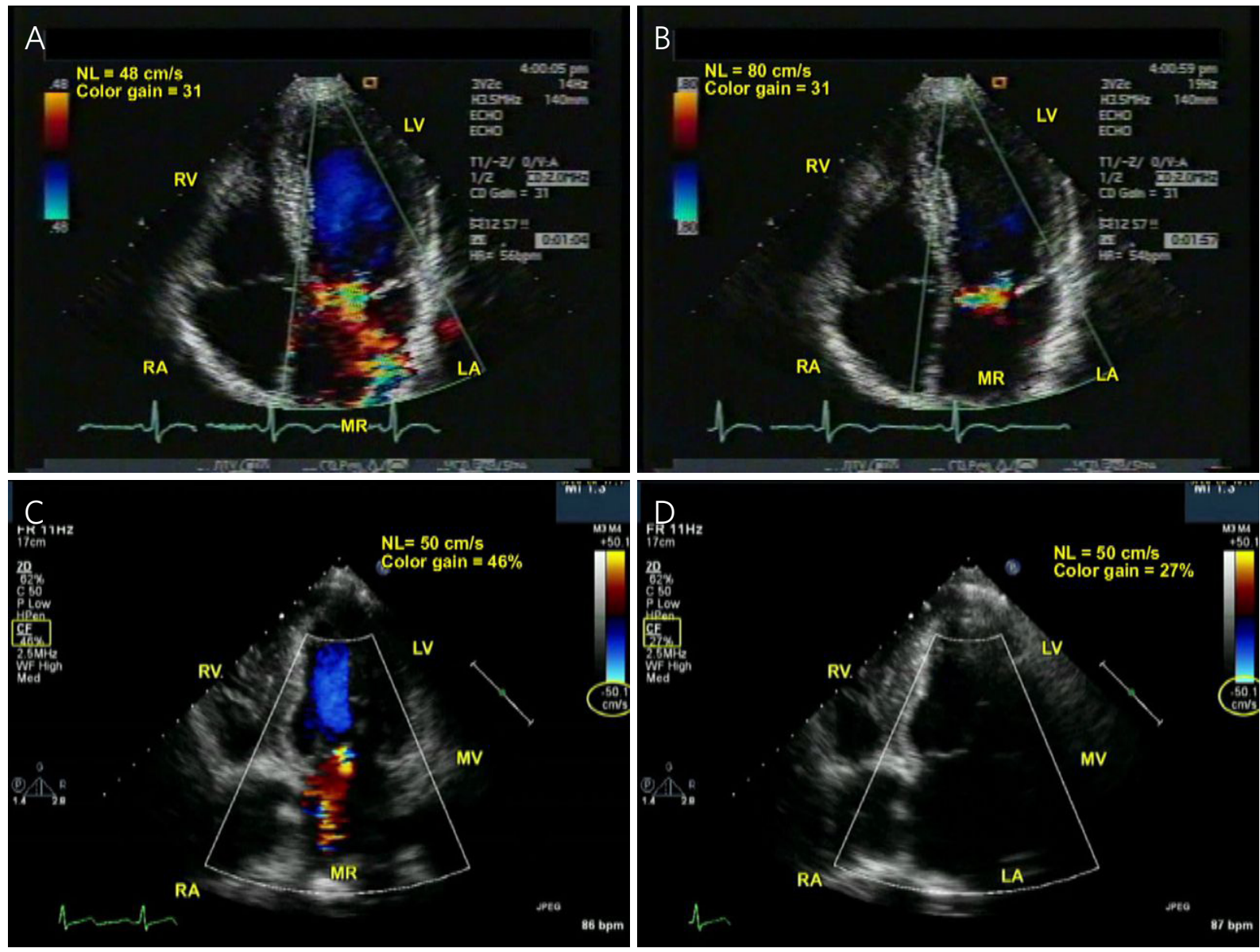

Figure 9. Two-dimensional transthoracic echocardiography. Apical four-chamber views. A, B: when the NL of $48 \mathrm{~cm} / \mathrm{s}$ ( $A$ ) was increased to $80 \mathrm{~cm} / \mathrm{s}$ (B) without moving the transducer and keeping the color Doppler gain constant at 31, the MR flow signals showed marked reduction in size in this patient with substantial MR; C, D: in another patient with substantial MR, reducing color Doppler gain from 46\% (C) to $27 \%$ (D) keeping the NL constant at $50 \mathrm{~cm} / \mathrm{s}$ and not moving the transducer resulted in complete disappearance of MR signals. Color gain is optimized by first increasing it till stationary artifactual echoes often extending beyond the LA walls appear and then decreasing it gradually till they just disappear. LA: left atrium; MR: mitral regurgitation; NL: Nyquist limit; RV: right ventricle; RA: right atrium; LV: left ventricle; MV: mitral valve. Reproduced with permission from Manjunath et al. ${ }^{[4]}$

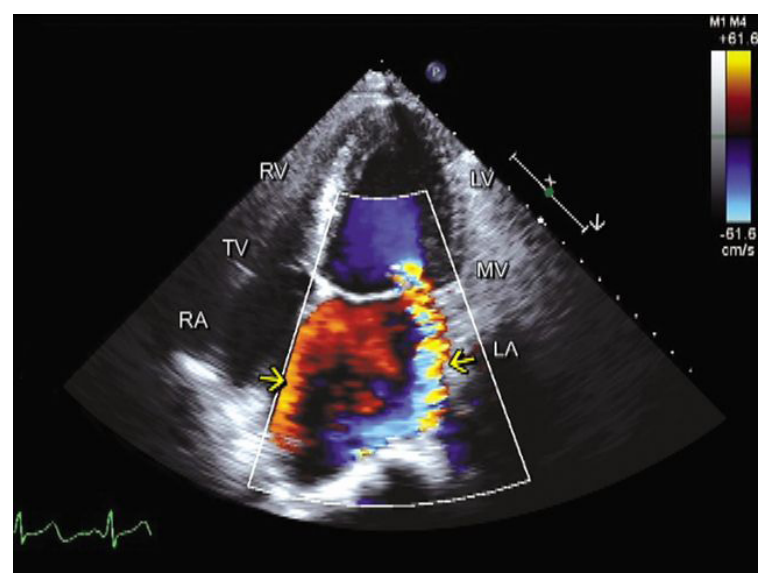

Figure 10. Two-dimensional transthoracic echocardiography in apical four-chamber view in a patient with severe MR. A relatively small size wall hugging turbulent eccentric MR jet (right arrow) consistent with mild MR is noted. If the laminar flow signals (red, left arrow) moving in the same phasic manner as the turbulent MR jet (to differentiate them from pulmonary venous inflow) are taken into account, MR severity would not be underestimated and would be correctly considered severe, as the combined turbulent and laminar flow signals virtually fill the whole LA. The laminar low velocity red flow signals also representing MR result from the high velocity turbulent flow signals striking the LA lateral wall resulting in marked reduction of their velocity. RV: right ventricle; RA: right atrium; LV: left ventricle; LA: left atrium; MV: mitral valve; MR: mitral regurgitation; TV: tricuspid valve. Reproduced with permission from Manjunath et al. ${ }^{[4]}$ 

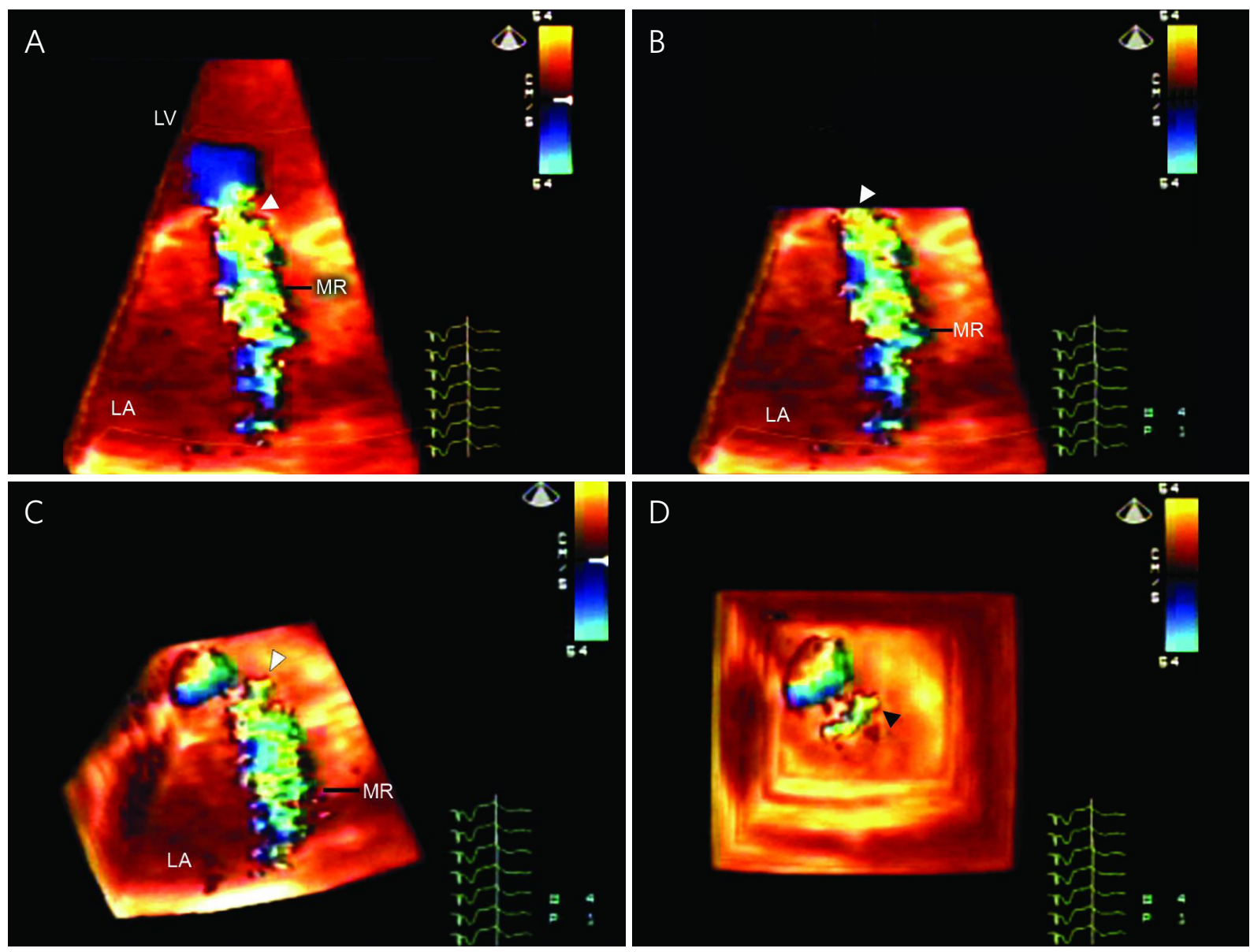

Figure 11. A-D: Live/real-time three-dimensional color Doppler transthoracic echocardiographic technique for assessment of vena contracta area. Three-dimensional color Doppler dataset showing MR (A) cropped from top to the level of the vena contracta (arrowhead, $B$ ) and tilted to view it en face (arrowheads in $C$ and $D$ ). The vena contracta area is then measured by planimetry. MR: mitral regurgitation. Reproduced with permission from Khanna et al. ${ }^{[30]}$

convergence zone, elevated mitral inflow E velocity $(>1.2 \mathrm{~m} / \mathrm{s})$ or systolic flow reversal into pulmonary veins are also useful for accurate assessment of MR severity in such cases of eccentric MR.

Due to several technical and hemodynamic limitations, CD should not be used alone to assess MR severity ${ }^{[2]}$. Therefore, an integrative approach using supportive spectral Doppler and 2D echocardiographic parameters is recommended when suspecting significant MR on CD. The utility of 3D CD in assessing MR severity is emerging because of its ability to view both the flow convergence zone and the vena contracta en face $^{[26]}$.

\section{VENA CONTRACTA}

The vena contracta width is the narrowest region of the MR jet that occurs at or immediately downstream of the regurgitant orifice. When assessed in $3 \mathrm{D}$ en face view, it represents the defect through which MR occurs or in other words the cross-sectional area of the effective regurgitant orifice area [Figures 11 and 12] $]^{[27]}$. For accurate measurement, $\mathrm{VC}$ in $2 \mathrm{D}$ imaging should be assessed in a zoom view perpendicular to the commissural line (e.g., the parasternal long axis or the apical 4-chamber view). In general, the $\mathrm{VC}$ is independent of flow rate or driving pressure ${ }^{[28]}$. Regardless of MR etiology, VC is a useful semiquantitative measure of MR severity in both central and eccentric jets ${ }^{[27]}$. It is recommended to average $\mathrm{VC}$ measurements over 2 to 3 beats using two orthogonal planes ${ }^{[22]}$. A VC width of $<3 \mathrm{~mm}$ indicates mild 


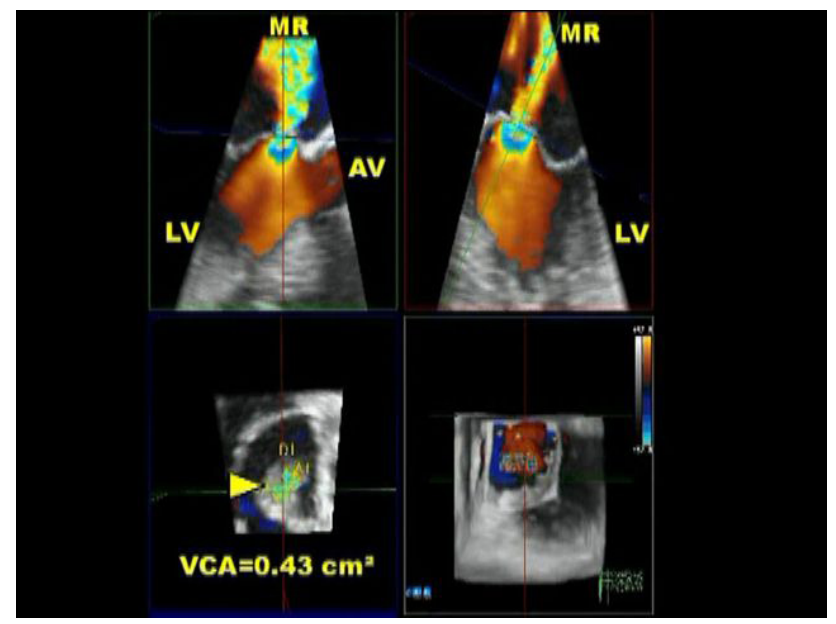

Figure 12. Live/real-time three-dimensional color Doppler transesophageal echocardiographic technique for assessment of VCA. The three-dimensional dataset is cropped at the level of the MR vena contracta (MR jet origin) and cropped to view it en face (arrowhead). VCA measured $0.43 \mathrm{~cm}^{2}$, indicative of severe MR. VCA: vena contracta area; LV: left ventricle; MR: mitral regurgitation; AV: aortic valve. Copyright with Navin C. Nanda

$\mathrm{MR}$, whereas a width of $>7 \mathrm{~mm}$ defines severe $\mathrm{MR}^{[22]}$. If multiple jets are noted, the widths of $\mathrm{VC}$ maybe additive. However, VC may underestimate MR severity in cases of multiple jets or if there is an elliptical regurgitant orifice. On the other hand, VC could overestimate MR severity if regurgitant jet is limited to early, mid or late systole ${ }^{[29]}$. Recently, emerging data show that 3D area measurements of VC as well as flow convergence may provide useful quantitative assessment of MR severity ${ }^{[30,31]}$.

\section{FLOW CONVERGENCE/PROXIMAL ISOVELOCITY SURFACE AREA}

Imaging the flow convergence region proximal to the regurgitant orifice is highly recommended for MR quantitation. Qualitatively, the presence of a large convergence zone indicates substantial MR [Figure 8]. Quantitatively, the flow convergence method can be used to quantify the regurgitant flow rate, which is used to calculate the EROA, RVol and regurgitant fraction $(\mathrm{RF})^{[32]}$. These are essential measures of lesion severity, volume overload and predict outcomes for patients with severe MR. The proximal isovelocity surface area (PISA) method is based on the continuity principle ${ }^{[33]}$. In any regurgitant lesion, blood flow accelerates towards the regurgitant orifice and creates concentric hemispherical isovelocity surfaces (shells) centered at the regurgitant orifice [Figure 13] ${ }^{[34]}$. The blood flow rate across all these hemispherical surfaces is constant and equal to the flow rate through the regurgitant orifice ${ }^{[33]}$. Color flow mapping provides the ability to visualize any one of these hemispheres that corresponds to certain aliasing velocity threshold. The apical 4-chamber view is recommended for optimal PISA measurements. The area of interest is optimized by lowering the image depth and shifting the Nyquist limit towards the direction of MR jet, i.e., down on TTE and up on TEE. To calculate the flow rate, multiply the aliasing velocity (Va) by $2 \pi \mathrm{r}^{2}$ (area of corresponding hemisphere), where $\mathrm{r}$ is PISA radius [Figure 14$]^{[4]}$. On the basis of regurgitant flow, EROA, $\mathrm{RVol}$ and RF can be calculated using standard formulas.

Regurgitant flow $=\mathrm{Va} \times 2 \pi \mathrm{r}^{2}$

EROA = Regurgitant flow/Peak velocity of MR

$\mathrm{RVol}=\mathrm{EROA} \times$ Velocity Time Interval of MR

$\mathrm{RF}=\mathrm{RVol} /$ stroke volume of regurgitant valve

A simplified method to calculate EROA is to measure PISA radius at Nyquist limit of $40 \mathrm{~cm} / \mathrm{s}$, assume peak MR jet velocity of $5 \mathrm{~m} / \mathrm{s}$, and then EROA $=\mathrm{r}^{2} / 2^{[17]}$. Regardless of MR etiology, EROA of $\geq 0.4 \mathrm{~cm}^{2}$ and/or RVol 


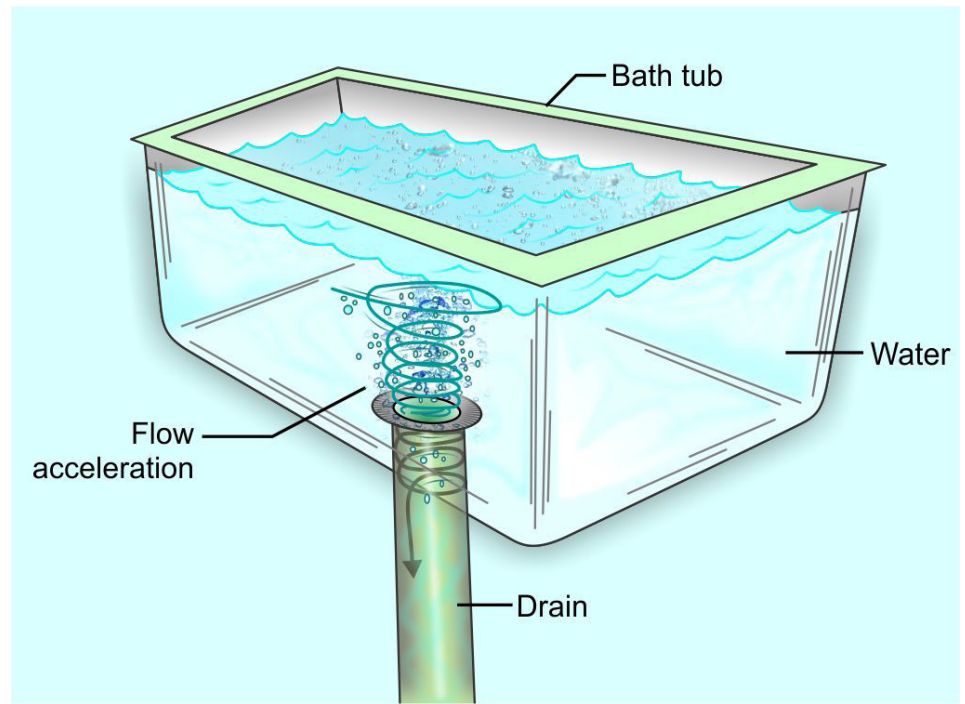

Figure 13. A simple example of the generation of flow acceleration can be shown by observing the draining of water from a household bathtub. Flow acceleration or a localized area of high velocity develops as the large body of water moves toward the "hole" or opening in the bottom of the tub, through which water flows into the drain. Adjacent to this "hole," the area of flow acceleration becomes smaller and tends to take the shape and size of the circular "hole" (vena contracta). Reproduced with permission from Kapur et al. ${ }^{[34]}$

\section{Flow convergence method}
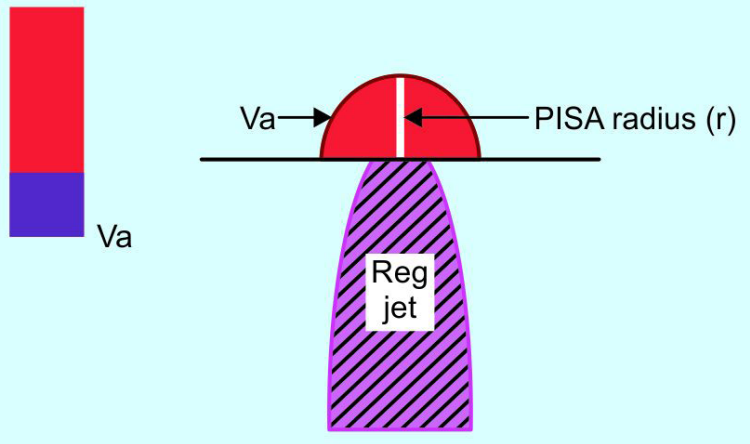

Reg flow $=2 \pi r^{2} \times V a$ $E R O A=$ Reg flow $/ \mathrm{PkV}_{\text {Reg }}$

Figure 14. Schematic depiction of the flow convergence or PISA method for quantitating valvular regurgitation. Va is the velocity at which aliasing occurs in the flow convergence toward the regurgitant orifice. PISA: proximal isovelocity surface area; EROA: effective regurgitant orifice area; PkV : peak velocity of the regurgitant jet determined by continuous wave Doppler; Reg flow: regurgitant flow; Reg jet: regurgitation jet. Reproduced with permission from Manjunath et al. ${ }^{[4]}$

$\geq 60 \mathrm{~mL}$ indicates severe MR [Table 2]. However, in secondary MR, EROA $\geq 0.2 \mathrm{~cm}^{2}$ and/or $\mathrm{RVol} \geq 30 \mathrm{~mL}$ is associated with worse outcomes ${ }^{[35]}$.

Similar to VC measurement, the PISA method could be misleading if multiple jets or noncircular regurgitant orifices present. It could be technically challenging to obtain accurate PISA measurements in cases of eccentric jets. A major problem in quantifying MR severity by the PISA method is the assumption that the flow convergence is hemispherical in shape, which is not the case in most patients with MR. Thus, EROA by PISA equation is not recommended in the presence of MV devices including MitraClip, because 
the assumption of hemispherical proximal flow conversion zone is even further disrupted by the device ${ }^{[1,14]}$. The use of 3D echocardiography could help overcome some of these limitations.

\section{SPECTRAL DOPPLER}

Spectral Doppler remains useful and provides important parameters for quantitative assessment of MR severity [Table 3]. Both the mitral to aortic TVI ratio of $>1.4$ and the systolic flow reversal into pulmonary veins are specific signs of severe MR. Similar to the quantitative volumetric method, the pulsed wave Doppler method is time-consuming and has several limitations ${ }^{[36]}$.

\section{ROLE OF STRESS TESTING}

Both in primary and secondary MR, exercise stress echocardiography can provide additional diagnostic and prognostic information in asymptomatic patients ${ }^{[37]}$. For patients with severe MR and equivocal symptoms, exercise testing can be useful in assessing symptomatic status and functional capacity. Inadequate increase in $\mathrm{LV}$ ejection fraction with exercise predicts worse postoperative LV function ${ }^{[38]}$. In secondary $\mathrm{MR}$, an increase in EROA $>0.13 \mathrm{~cm}^{2}$ during exercise is associated with worse cardiovascular outcomes $^{[37]}$. Currently, there is no role for pharmacological stress echocardiography in evaluation of MR severity.

\section{CONCLUSION}

$2 \mathrm{D}$ echo imaging is the modality of choice for evaluating the etiology and mechanism of MR and associated lesions. MR severity in real world practice is semi-quantitatively assessed by eyeballing the proportion of the LA area occupied by the regurgitant jet on $2 \mathrm{D} /$ color Doppler imaging. This is supplemented by linear measurements of flow convergence and VC. When MR appears moderately severe or severe by these methods and intervention, where MitraClip is a consideration, more comprehensive and complicated quantitative echo methods, which may include 3D imaging, are used.

\section{DECLARATIONS}

\section{Authors' contributions}

Read and approved the manuscript: Smer A, Nanda NC, Akdogan RE, Elmarzouky ZM, Dulal S

\section{Availability of data and materials}

Not applicable.

\section{Financial support and sponsorship}

None.

\section{Conflicts of interest}

All authors declared that there are no conflicts of interest.

\section{Ethical approval and consent to participate}

Not applicable.

\section{Consent for publication}

Not applicable.

\section{Copyright}

(c) The Author(s) 2020. 


\section{REFERENCES}

1. Zoghbi WA, Adams D, Bonow RO, Enriquez-Sarano M, Foster E, et al. Recommendations for noninvasive evaluation of native valvular regurgitation: a report from the american society of echocardiography developed in collaboration with the society for cardiovascular magnetic resonance. J Am Soc Echocardiogr 2017;30:303-71.

2. Nishimura RA, Otto CM, Bonow RO, Carabello BA, Erwin JP, et al. 2014 AHA/ACC guideline for the management of patients with valvular heart disease: a report of the american college of cardiology/american heart association task force on practice guidelines. J Am Coll Cardiol 2014;63:e57-185.

3. O'Gara P, Sugeng L, Lang R, Sarano M, Hung J, et al. The role of imaging in chronic degenerative mitral regurgitation. JACC Cardiovasc Imaging 2008;1:221-37.

4. Manjunath CN, Moorthy N, Bowen L, Nanda NC. Chapter 38, Echocardiographic assessment of mitral valve disease. In: Nanda NC, editor. Comprehensive textbook of echocardiography. 2nd ed. New Delhi, India: Jaypee Brothers Medical Publishers; 2020. pp. 848-900.

5. Nishimura RA, Otto CM, Bonow RO, Carabello BA, Erwin JP, et al. 2017 AHA/ACC focused update of the 2014 AHA/ACC guideline for the management of patients with valvular heart disease: a report of the american college of cardiology/american heart association task force on clinical practice guidelines. J Am Coll Cardiol 2017;70:252-89.

6. Agricola E, Oppizzi M, Pisani M, Meris A, Maisano F, et al. Ischemic mitral regurgitation: Mechanisms and echocardiographic classification. Eur J Echocardiogr 2008;9:207-21.

7. Tibayan FA, Wilson A, Lai DT, Timek TA, Dagum P, et al. Tenting volume: three-dimensional assessment of geometric perturbations in functional mitral regurgitation and implications for surgical repair. J Heart Valve Dis 2007;16:1-7.

8. Ito K, Abe Y, Takahashi Y, Shimada Y, Fukumoto H, et al. Mechanism of atrial functional mitral regurgitation in patients with atrial fibrillation: a study using three-dimensional transesophageal echocardiography. J Cardiol 2017;70:584-90.

9. Saurav A, Smer A, Azzouz MS, Abuissa H, Hunter C. Impact of non-apical right ventricular lead placement on left ventricular reverse remodeling and inter-ventricular dys-synchrony in cardiac resynchronization therapy: a meta-analysis. J Card Fail 2015;21:S43.

10. Kotler MN, Mintz GS, Parry WR, Segal BL. M mode and two dimensional echocardiography in mitral and aortic regurgitation: Pre- and postoperative evaluation of volume overload of the left ventricle. Am J Cardiol 1980;46:1144-52.

11. Pierard LA, Henri C, Magne J, Arora G. Chapter 39, Mitral regurgitation. In: Nanda NC, editor. Comprehensive textbook of echocardiography. 2nd ed. New Delhi, India: Jaypee Brothers Medical Publishers; 2020. pp. 901-17.

12. Feldman T, Kar S, Elmariah S, Smart SC, Trento A, et al. Randomized comparison of percutaneous repair and surgery for mitral regurgitation: 5-year results of EVEREST II. J Am Coll Cardiol 2015;66:2844-54.

13. Pleger ST, Schulz-Schönhagen M, Geis N, Mereles D, Chorianopoulos E, et al. One year clinical efficacy and reverse cardiac remodelling in patients with severe mitral regurgitation and reduced ejection fraction after MitraClip implantation. Eur J Heart Fail 2013;15:919-27.

14. O'Gara PT, Grayburn PA, Badhwar V, Afonso LC, Carroll JD, et al. 2017 ACC expert consensus decision pathway on the management of mitral regurgitation: a report of the american college of cardiology task force on expert consensus decision pathways. J Am Coll Cardiol 2017;70:2421-49.

15. Abuzaid A, Smer A, Nanda N, Salama AY. 3-usefulness of three-dimensional echocardiography in clinical practice. Sri Lankan J Cardiol 2019;1:13-23.

16. Chaliki HP, Nishimura RA, Enriquez-Sarano M, Reeder GS. A simplified, practical approach to assessment of severity of mitral regurgitation by doppler color flow imaging with proximal convergence: validation with concomitant cardiac catheterization. Mayo Clin Proc 1998;73:929-35.

17. Khanna D, Miller AP, Nanda NC, Ahmed S, Lloyd SG. Transthoracic and transesophageal echocardiographic assessment of mitral regurgitation severity: usefulness of qualitative and semiquantitative techniques. Echocardiography 2005;22:748-69.

18. Grayburn PA, Carabello B, Hung J, Gillam LD, Liang D, et al. Defining "severe" secondary mitral regurgitation: emphasizing an integrated approach. J Am Coll Cardiol 2014;64:2792-801.

19. Stone GW, Lindenfeld J, Abraham WT, Kar S, Lim DS, et al. Transcatheter mitral-valve repair in patients with heart failure. N Engl J Med 2018;379:2307-18.

20. Obadia JF, Messika-Zeitoun D, Leurent G, Iung B, Bonnet G, et al. Percutaneous repair or medical treatment for secondary mitral regurgitation. N Engl J Med 2018;379:2297-306.

21. Grayburn PA, Sannino A, Packer M. Proportionate and disproportionate functional mitral regurgitation: a new conceptual framework that reconciles the results of the MITRA-FR and COAPT trials. JACC Cardiovasc Imaging 2019;12:353-62.

22. Lancellotti P, Tribouilloy C, Hagendorff A, Popescu BA, Edvardsen T, et al. Recommendations for the echocardiographic assessment of native valvular regurgitation: an executive summary from the european association of cardiovascular imaging. Eur Heart J Cardiovasc Imaging 2013;14:611-44.

23. Helmcke F, Nanda NC, Hsiung MC, Soto B, Adey CK, et al. Color doppler assessment of mitral regurgitation with orthogonal planes. Circulation 1987;75:175-83.

24. Lin BA, Forouhar AS, Pahlevan NM, Anastassiou CA, Grayburn PA, et al. Color doppler jet area overestimates regurgitant volume when multiple jets are present. J Am Soc Echocardiogr 2010;23:993-1000.

25. Chao K, Moises VA, Shandas R, Elkadi T, Sahn DJ, et al. Influence of the coanda effect on color doppler jet area and color encoding. in vitro studies using color doppler flow mapping. Circulation 1992;85:333-41.

26. Tan TC, Zeng X, Jiao Y, Wang L, Wei Q, et al. Three-dimensional field optimization method: clinical validation of a novel color doppler method for quantifying mitral regurgitation. J Am Soc Echocardiogr 2016;29:926-34.

27. Hall SA, Brickner ME, Willett DL, Irani WN, Afridi I, et al. Assessment of mitral regurgitation severity by doppler color flow mapping of 
the vena contracta. Circulation 1997;95:636-42.

28. Baumgartner H, Schima H, Kuhn P. Value and limitations of proximal jet dimensions for the quantitation of valvular regurgitation: an in vitro study using doppler flow imaging. J Am Soc Echocardiogr 1991;4:57-66.

29. Biner S, Rafique A, Rafii F, Tolstrup K, Noorani O, et al. Reproducibility of proximal isovelocity surface area, vena contracta, and regurgitant jet area for assessment of mitral regurgitation severity. JACC Cardiovasc Imaging 2010;3:235-43.

30. Khanna D, Vengala S, Miller AP, Nanda NC, Lloyd SG, et al. Quantification of mitral regurgitation by live three-dimensional transthoracic echocardiographic measurements of vena contracta area. Echocardiography 2004;21:737-43.

31. Zeng X, Levine RA, Hua L, Morris EL, Kang Y, et al. Diagnostic value of vena contracta area in the quantification of mitral regurgitation severity by color doppler 3D echocardiography. Circ Cardiovasc Imaging 2011;4:506-13.

32. Enriquez-Sarano M, Avierinos JF, Messika-Zeitoun D, Detaint D, Capps M, et al. Quantitative determinants of the outcome of asymptomatic mitral regurgitation. N Engl J Med 2005;352:875-83.

33. Bargiggia GS, Tronconi L, Sahn DJ, Recusani F, Raisaro A, et al. A new method for quantitation of mitral regurgitation based on color flow doppler imaging of flow convergence proximal to regurgitant orifice. Circulation 1991;84:1481-9.

34. Kapur K, Fan PH, Nanda NC. Chapter 18, Conventional and color Doppler in the assessment of prosthetic valve function. In: Nanda NC, editor. Textbook of color Doppler echocardiography. Philadelphia: Lea \& Febiger; 1989. pp. 198-210.

35. Lancellotti P, Troisfontaines P, Toussaint AC, Pierard LA. Prognostic importance of exercise-induced changes in mitral regurgitation in patients with chronic ischemic left ventricular dysfunction. Circulation 2003;108:1713-7.

36. Dujardin KS, Enriquez-Sarano M, Bailey KR, Nishimura RA, Seward JB, et al. Grading of mitral regurgitation by quantitative doppler echocardiography: Calibration by left ventricular angiography in routine clinical practice. Circulation 1997;96:3409-15.

37. Lancellotti P, Moura L, Pierard LA, Agricola E, Popescu BA, et al. European association of echocardiography recommendations for the assessment of valvular regurgitation. part 2: mitral and tricuspid regurgitation (native valve disease). Eur J Echocardiogr 2010;11:307-32.

38. Leung DY, Griffin BP, Stewart WJ, Cosgrove DM, Thomas JD, et al. Left ventricular function after valve repair for chronic mitral regurgitation: predictive value of preoperative assessment of contractile reserve by exercise echocardiography. J Am Coll Cardiol 1996;28:1198-205. 